\title{
Qualitative Study of Existing Research Techniques on Wireless Mesh Network
}

\author{
Naveen T.H \\ Research Scholar \\ Visvesvaraya Technological University \\ Belagavi, \\ Karnataka, \\ India
}

\author{
Vasanth $\mathrm{G}$ \\ Prof \& Head \\ Dept. of Computer Science \& Engineering \\ Government Engineering College, \\ Krishnarajpet,Mandya District, \\ Karnataka, India
}

\begin{abstract}
Wireless Mesh Network (WMN) is one of the significant forms of the wireless mesh network that assists in creating highly interconnected communication node. Since a decade, there have been various studies towards enhancing the performance of WMN which is successful to a large extent. However, with the upcoming technology of pervasive and dynamic networks WMN suffers from various routing issues, Quality-of-Service (QoS) issue, channel allocation, sustainability of routes which makes the theory contradicting when considering for real-world challenges in wireless networks. This paper, therefore, briefs about fundamental information of WMN followed by a discussion of existing research trends and existing research techniques. Finally, the paper also discusses the open research issues after reviewing the existing research techniques.
\end{abstract}

Keywords-Access Points; Channel Allocation; Internet Access; Routing Problems; Wireless Mesh Network; QoS

\section{INTRODUCTION}

In last few years, the energy consumption, as well as cost of the networking devices, is increasing rapidly. Energy is always a matter of concern for any form of wireless network. It is because the optimal residual energy of a node and its higher retention capability can increase the network longevity [1] [2]. In the area of the wireless network, Wireless Mesh Network (WMN) is one of the frequently selected topics of research owing to its increasing number of research problems [3][4][5]. Although WMN seems to have an easier implementation, there are certain sets of common problems which have been addressed by most of the researchers. The first problem in conventional WMN is to perform the selection of a precise radio technique over physical layer [6]. At present, the alternatives of such techniques are Multiple-input Multipleoutput (MIMO), Ultra Wide Band (UWB), Code Division Multiple Access (CDMA), etc. [6]. For nodes to work effectively, it is also required to have the faster frequency switching capability. The second problem in WMN is that conventional contention-based methodologies are never enough to enhance the fairness or channel allocations [7]. In order to maintain a mesh topology, it is required for a node to cost-effectively adopt the multiple physical channels, which at present is still an open problem from the viewpoint of channel assignment. WMN is also increasing investigated on $4 \mathrm{G}$ and $5 \mathrm{G}$ network [8][9][10]. It should be noted that such faster data transmission will require utilizing smart antenna in $\mathrm{WMN}$, which is still an open issue in WMN. It is because the selection of the better version of MAC protocol is still an open problem [11]. Apart from this design and development of routing mechanism in order to accomplish QoS, robustness in communication, reliability, and efficiency is still an open challenge in WMN. The third problem of WMN is poor performance of the transport protocol that still encounters issues in utilizing network resources in order to perform channel allocation fairly. Although, TCP is the most frequently adopted communication protocol over the internet but it cannot be used for WMN. From the application viewpoint, there are challenges too. It is a very challenging task for WMN for offering internet connectivity with retention of QoS [12][13][14][15]. Hence, topological problems along with deployment problems persist over WMN even in recent times. The fourth problem of WMN is scheduling or provisioning problems. The biggest challenge in WMN is that if the number of user or nodes keeps on increasing, it is very difficult to optimize the channel capacity. For this reason, the communication performance highly degrades to a larger extent those results in declination of channel capacity. Although such problems could be possibly overcome by adding new gateway nodes in the adjacent transmission zone, it gives rise to another challenging issue i.e. how to identify the location of repeaters or base station to be fixed? The sole purpose will be obviously to increase the channel capacity and to offer equal QoS to all the connected users. As such forms of a network are connected to each other using internet access; hence it is imperative that dealing with the communication-based requirement will be one of the toughest tasks in WMN. As WMN can be looked upon as an easier network implementation with flexibility to accommodate various numbers of nodes.

Hence, this paper reviews some of the existing techniques to circumvent such problems related to communication and looks for the open research problems in it. This paper describes the routing mechanisms for the mesh network and also the existing research work in it. The Sectional organization is as follows. Section II briefly highlights the fundamental information about WMN followed by the discussion of present research trends in Section III. Discussion of existing research techniques towards varied problems in WMN is carried out in Section IV followed by highlighting of open research issues in Section V. Finally, the conclusion and future work is briefed in Section VI. 


\section{WIRELESS MESH NETWORK}

Basically, a WMN can be defined as an interconnected radio nodes using mesh topology. The formation of the WMN is done by a mesh access point, a mesh user, and gateway. A good example to understand mesh clients are smart phones, personal digital assistant while the mesh access point transmits the traffic towards the gateway. In WMN, it is not necessary for the gateway node to be connected to World Wide Web. In WMN, a mesh cloud is defined as the transmission zones of communication nodes that combine work as a single network. It is feasible for the mesh cloud to have access to such mesh cloud that finally forms a radio network that provides redundancy and is highly trustworthy. Interestingly, failure of a single node doesn't affect the communication process as other nodes find an alternative way to perform communication. WMN is also found to be working with existing IEEE standards e.g. 802.15, 802.11, 802.16, etc. Application of mesh network can be easily found in US military [16], smart energy system [17], satellite phones [18], etc. The WMN is an upcoming innovation that can convey Internet broadband access, remote access, and system availability for network system connectivity among administrators and clients at low expenses. It is correspondence systems that have progressively pulled in Internet Service Providers (ISPs) due to its fast developing and evolution of remote advancements. WMN is a promising innovation in giving high data transfer capacity system scope. WMNs will incredibly help the clients to be constantly online anyplace at whatever time by associating with remote cross section switches [19]-[24].

\section{A. WMN architecture}

Designing WMN architecture is an initial move towards giving high-speed Internet connectivity over a particular scope region. WMN uses Wireless Mesh Routers (WMRs) and Mesh Clients (MCs) for transmitting the data packet in a multi-hop pattern, while the network devices e.g. gateway have the lesser amount of adaptability as well as supportability towards the backbone network. To outline more, it is comprised of different forms of the communicating nodes that can interact with each other [22][23]. Middle of the routing nodes manages the signal quality, as well as forward bundles in the interest of different nodes. Additionally, it gives high-transmission capacity Internet get to and offers a minimal effort and adaptable organization. The foundation that backings a WMN are a remote cross section switch system, or Backbone Wireless Mesh Network (BWMN). BWMN gives Internet network to MCs in a multi-bounce style. MCs can get to the Internet through BWMN shaped by Wireless Mesh Routers (WMRs). A common WMN is delineated in Fig.1.There are three sorts of nodes in a WMN: WMN router, WMN gateway, and WMN client. The clients of WMN are the end-client gadgets, for example, portable PCs, PDAs, advanced cells, and so on, that can get to the system for utilizing applications like email, VoIP, diversion, area discovery, and so forth. These gadgets are thought to be versatile; they have constrained force, they may have the directing ability, and might constantly be associated with the system. WMN switches are in the system to course the system movement. They can't end nor start the movement. The switches have the restriction in portability, and they have dependable qualities. Transmission power utilization in cross section switches is low, for multi-jump interchanges system. Moreover, the Medium Access Control (MAC) convention in a cross section switch underpins various channels and numerous interfaces to empower adaptability in a multi-bounce network environment. WMN portals are switches with wired base/the Internet. Since the passages in WMNs have various interfaces to associate with both wired and remote systems, they are costly. Consequently, there is a couple of number of WMN passages in the system. Also, their arrangement significantly affects the execution of the system. Fig. 1 shows the WMN architecture with Mesh Router (MR), GWN (Gateway Node), WC (Wireless Client), AP (Access Point), BS (Base Station), and PC (Personal Computer).

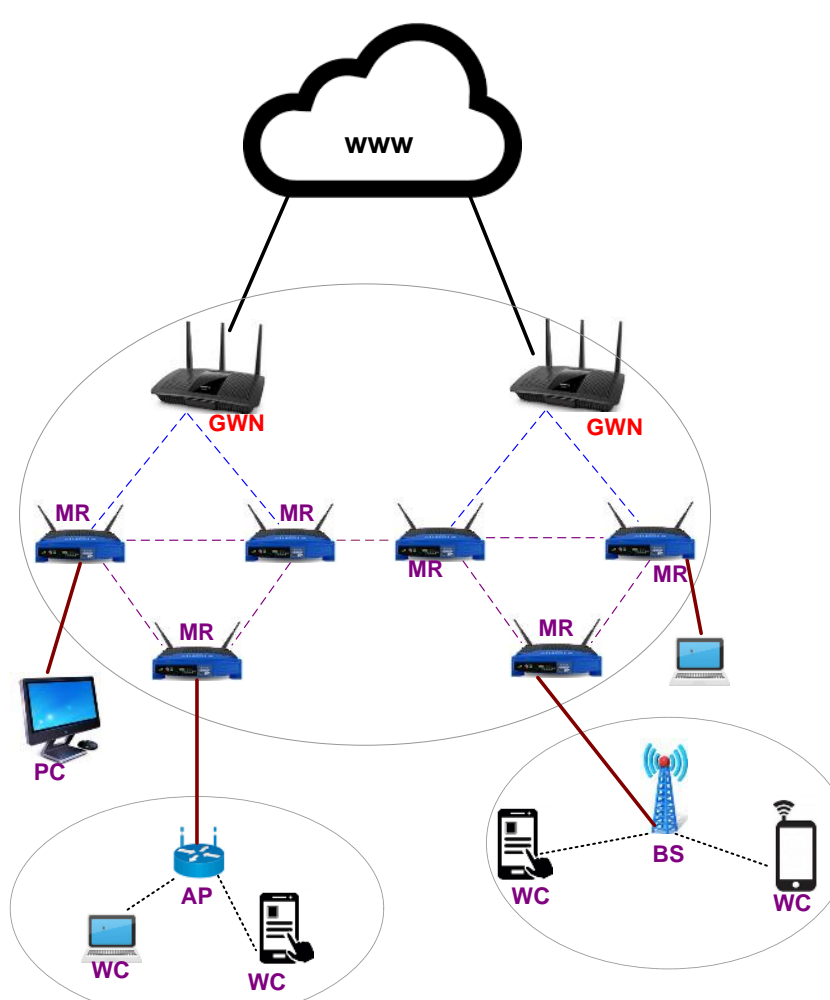

Fig. 1. WMN architecture

\section{B. Classification of WMN}

The connectivity based WMNs can be classified as sorts of the different system components, which are either Point to Multi-Point (MPM), Multi-Point (PTM), Point To Point (PTP), or Multi-Point to systems. The complete scientific categorization of this grouping appears in Fig.2. It is believed that PTP forms of the WMN are highly trusted worthy and offer very easier implementation of the wireless network. It basically consists of two communicating nodes (or radio) along with antenna with high gain in order to accomplish highquality links. Such links are used for applications that demand maximized communication performance with higher speed data transmission. Unfortunately, PTP forms lack scalability and also suffers from lower adaptability. PTM form of network applies star topology to support both single and dual direction transmission. It normally uses the omnidirectional antenna for facilitating uplink transmission, and it uses the antenna with 
high gain for supporting downlink transmission. Uses of PTM network are highly suitable for clients requiring high-speed data transmission without much focus on channel capacity. It is also used in backhaul operation. Although PTM networks are scalable to the moderate extent, it lacks reliability as well as adaptability. MTM network is meant for overcoming the flaws of PTM network i.e. to offer the higher degree of adaptability, reliability, and scalability. It is also suitable for large-scale network deployment. In MTM, the communicating devices are inter-connected with various forms of network nodes e.g. switches and routers. The increase in node number also has a positive effect on energy conservation. The utilization of these three forms of the WMN depends on the types of the application and networking demands of the clients.

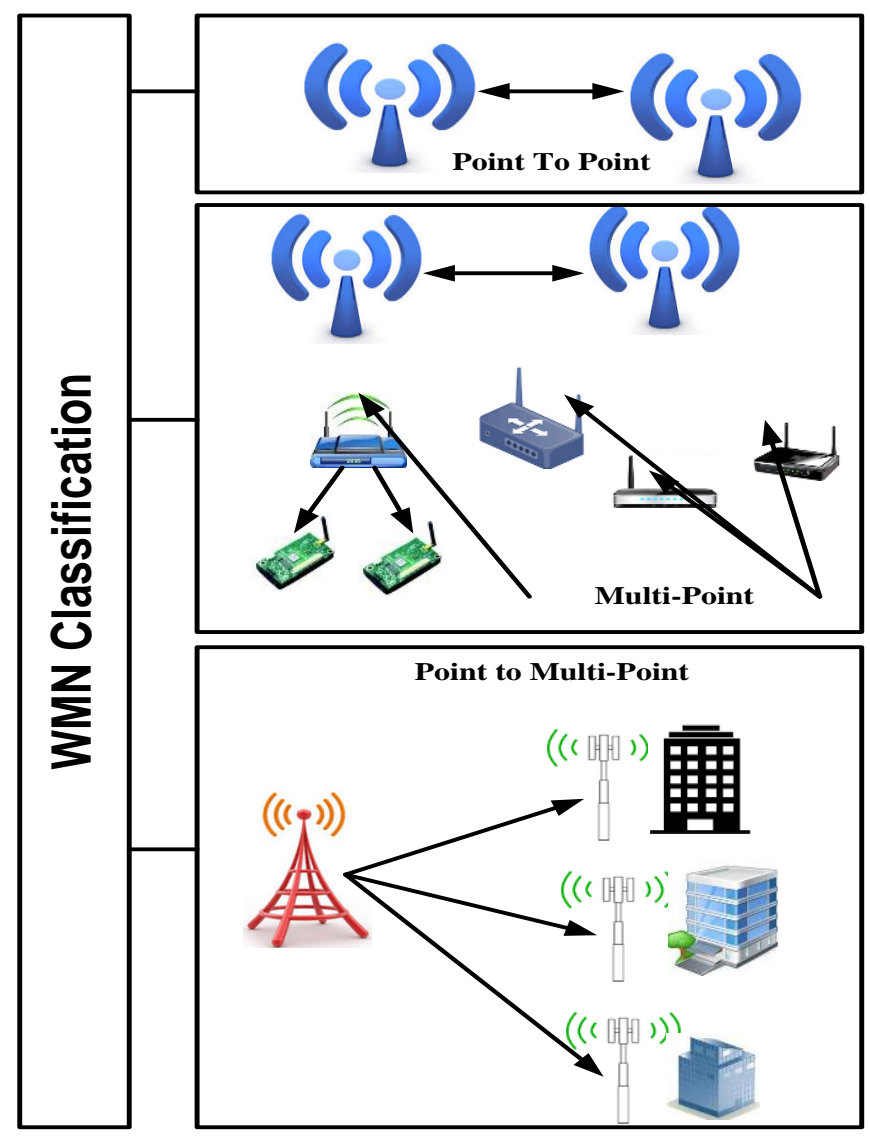

Fig. 2. WMN classification

\section{Existing Standards for WMN}

Numerous research organizations have continuous exploration ventures on different parts of WMN including energy management, standards, applications, and administrations. Some of the standards for WMN are described below.

- IEEE 802.11s Wi-Fi Mesh: Presently the IEEE 802.11 family is the adequate standard. Developed Service Set (DSS) and Wireless Distribution System are characterized in IEEE 802.11s for applying multibounce network methods and giving a convention to auto-arranging ways between WMRs. 802.11s has three fundamental parts viz. i) Network Portal (NP): goes about as a passage to different systems, ii) Network STA (station): goes about as a switch to hand-off edges hop by hop, and iii) Network AP (Access Point): gives handing-off capacities and also the availability administrations for customers.

- IEEE 802.15.1 Bluetooth: Bluetooth is the business name of this standard, and it is practiced on Wireless Personal Area Networks (WPANs). The task group is designed based on Bluetooth concept, and it correctly represents and specifies Media Access Control (MAC) and physical layer (PHY) for different forms of wireless transmission with either stationary or mobile nodes. There are two conceivable cross section topologies in WPAN network systems: full work topology or fractional lattice topology. A full work topology utilizes direct association plan. It implies that every remote node is associated specifically with every other node. Then again, some remote nodes are associated with all others while some are associated just to the remote hubs which hand-off the information.

- IEEE 802.15.4 Zigbee: Zigbee was first developed by Motorola. In Wireless Personal Area Network (WPAN) standard there are two association courses of action, single-bounce, and multi-jump association. Zigbee can bolster network topology by characterizing an organizer. The facilitator is capable of designing the system topology in multi-jump style. It is exceptionally appropriate for Wireless Sensor Mesh Networks (WSMNs).

- IEEE 802.16 WiMAX: This is a remote correspondence standard for Metropolitan Area Networks (MANs) which plans to give Mobile Multi-hop Relay (MHR) usefulness. This will permit sending multi-bounce network topology in WiMAX utilizing some WiMAX base stations to fill in as transfer stations. Setting up a multi-hop network topology gives a cost effective approach. The IEEE $802.16 \mathrm{j}$ standard was affirmed on May 2009 as a revision to the IEEE 802.16-2009 standard.

\section{Significances of Wireless Mesh Network:}

The following are uses of WMNs: -

1) Self-Organizing and Configuring: WMNs are adaptable in system design and not rely on upon the execution and the conventions. Self-mending and self-arranging are the WMNs highlights. This, diminishes the set-up time and support cost. Aside from this, it upgrades system execution. Because of these elements, the system administration suppliers can change, grow, and adjust the system as expected to achieve end clients requests.

2) Low Deployment Cost: Mesh switches are remote and they can benefit in multi-hop transmission. Therefore, utilizing remote switches as a part of substantial territories are less expensive contrasted with single jump switches/access focuses that they have wired associations. Ordinarily, because of wired associations those are more costly, in addition to quicker establishment and upkeep prompts a lower operation cost. 
3) Enhanced Reliability: One of the potential features of mesh network is its non-dependability of any specific node. This will mean that in case of any node failures, the communication is still facilitated by other inter-connected nodes. The system also introduced various data exchange mechanism to minimize the bottlenecks in congested zone of the system as well. This additionally permits the activity burdens to be adjusted in the system. The technique also permits various load balancing schemes in order to sustain heavy traffic condition.

4) Scalability: In conventional remote systems, when number of communication nodes increases, the system execution will be influenced to a large extent. Yet, in WMNs, expanding the quantity of communication nodes will build transmission limit for better load adjusting and backup ways to go. For the most part, the nearby bundles (created in customers of cross section switch) run quicker contrasted with packets (produced in two or more hops away) from the neighbors.

5) Interoperability: WMN has a hybrid design which is good with existing benchmarks, for example, WiMAX, Cellular, Wi-Fi, Zigbee, Vehicular, etc. Hence, it is appealing for incremental usage and reuse of existing base station. All innovations specified above, are capable or will be capable soon to arrange a WMN and perform communication with every other. The vast majority of fundamental changes required in systems to empower them speaks with others; can expand the present benchmarks to keep up interoperability [25].

\section{E. Routing conventions for WMN}

The routing mechanisms generally derived as proactive, reactive and hybrid type. Proactive methodology performs as established, wired network system. Routers ensure that no less than one way reaches to any goal. Then again, receptive conventions designate the way if just there is a packet that will be sent to the goal. On the other hand, a communication nodes do not have a packet to send to a specific goal; then communication nodes does not ask for a way to this goal. The routing for the WMNs can be taken as four main types like Adhoc based, Controlled flooding based, traffic aware based and opportunistic routing protocol [26][27].

- Ad-hoc Based Routing: Usage of an ad-hoc network is quite higher in WMN. It has good supportability of assisting in routing over dynamic topologies in WMN.

- Controlled Flooding-based Routing: These are used to reduce the control cost. In this, the point is to surge the system unnecessary as loads of association in remote systems happens between close communications nodes. Hence, it is not important to send control packets to inaccessible communication nodes as often as close nodes. Another method for lessening overhead is constraining the quantity of nodes which are in charge of flooding.

- Traffic-Aware based Routing: Traffic-based routing methods consider WMNs general traffic matrix. In this, the ad-hoc on demand distance vector-spreading over tree adjusts AODV from specially appointed systems.
In this routing, the gateway requires current way information from each communication nodes in the system to upgrade directing table.

\section{PRESENT RESEARCH TRENDS}

In order to understand the present research trend, we investigate the manuscript publication towards different forms of wireless network published in the duration of 2010 to till date.

TABLE. I. SUMMARY OF THE PRESENT RESEARCH TREND [CF: CONFERENCE, J: Journal, S: StANDARD, EAA: EARly ACCESS ARTicle, B: BOOKS, C: COURSE]

\begin{tabular}{|l|l|l|l|l|l|l|}
\hline Keyword & CF & J & S & EAA & B & C \\
\hline WMN & 3641 & 494 & 22 & 12 & 9 & 2 \\
\hline WSN & 39332 & 6921 & 365 & 60 & 7 & 1 \\
\hline WLAN & 12650 & 2192 & 157 & 40 & 5 & 4 \\
\hline $\begin{array}{l}\text { Cellular } \\
\text { Network }\end{array}$ & 788 & 23 & 0 & 4 & 0 & 0 \\
\hline $\begin{array}{l}\text { Adhoc } \\
\text { Network }\end{array}$ & 13042 & 3469 & 222 & 34 & 31 & 4 \\
\hline
\end{tabular}

From Table 1 , it is very much clear that there are very less work being carried out toward WMN as compared to other frequently used wireless network e.g. Wireless Sensor Network (WSN), Wireless Local Area Network (WLAN), cellular network, and adhoc network. Apart from this, we also observed the following trends:

- Energy Efficiency: There are approximately 70 journal pertaining to energy problems in WMN, which is quite less as there are various energy-modeling practices in wireless networks.

- Security: Security is received less attention with 46 journals whereas there are massive set of security algorithms.

- Routing: Routing has received massive attention as there are 214 journal and 1711 conference papers. Hence, there is a high progress made for communication protocols.

- Channel Assignment: Studies towards channel assignment has received the lowest research attention as can be seen from only 33journal.

- Optimization: Although, there are 76 journals towards optimization techniques in $\mathrm{WMN}$, it is very less in number as there are large numbers of optimization techniques in recent times.

\section{EXISTING RESEARCH TECHNIQUES}

This section discusses the existing research work towards wireless mesh network. It was noticed that in recent times, the prime emphasis was over the routing schemes in WMN. These schemes act as an enhancement towards the conventional communication protocols. Al-Saadi et al. [28] have addressed the communication problems in heterogeneous WMN with an objective of accomplishing better quality-of-service. The uniqueness of the study is that it has integrated current used $4 \mathrm{G}$ standards with IEEE 802.11 in order to incorporate cognitive principles over routing. The simulated study outcome was 
found to offer increased transmission capacity with enhanced capability to transmit the packet over longer routes. Study towards enhancing the throughput in WMN was carried out by Ashraf [29]. The authors have addressed the problem of determining a capacity for augmentation purpose in WMN. For this, a mesh network is designed with the single radio with the single channel with a target of achieving maximum traffic flow. The authors have also used mixed-integer linear programming with the greedy approach to finding increased throughput on various network topologies (both single and multiple). Routing techniques also directly affect the energy factor within the nodes and so is channel allocation mechanism. Although, there are various channel allocation techniques in wireless network but very few effective ones for WMN. Hence, Avallone et al. [30] have addressed the problem of integrated allocation of a channel as well as enhancing the routing operation. The authors have used the heuristic-based approach that allows least amount of energy to be allocated to each channel with multi-radio networks. Problems of accomplishing high throughput in WMN was also carried out by Chakraborty et al. [31] using an opportunistic approach. The primary problem addressed in this work is to countermeasure the exposed or latent node issues in WMN. The technique uses block acknowledgment as well as aggregation of data frames. For better outcomes, conventional collision avoidance techniques along with Carrier Sensing technology are used. Fadlullah et al. [32] have used the case study of solar power in WMN for checking out its effectiveness in power harvesting mechanism. A similar study considering problems of energy and throughput was seen in the work of $\mathrm{Li}$ et al. [33]. The major problems discussed the authors are instability and inadequacy of power supply owing to various forms of dynamics in WMN. The technique has presented both online and offline evaluation scheme for energy efficiency considering anticipated evaluation of incoming traffic. The position of the access points a significant role in routing process of WMN. Lin et al. [34] have presented a technique where optimization technique of simulated annealing is used over various priority constraints in WMN. Implemented over multiple cases of grids, the evaluation is continued for assessing the fitness function with respect to increasing rounds. Apart from access point position, the interface also affects the routing performance in WMN. One such work was carried out by Mansoori et al. [35] most recently where a different number of interfaces have been used over WMN with different and customized channel capacity to meet the transmission demands of various users in the network. Roh et al. [36] have presented a technique that performs allocation of the channel along with scheduling of routes in order to address the problem associated with controlling the rate. The authors have used Bender's decomposition algorithm to find the simulated outcome with optimized communication performance in a network. Yu et al. [37] have presented a technique that ensures quality of service and enhance the utilization technique of channel capacity.

The author Draves et al. [38] have defined significant study for $\mathrm{WMN}$, radio network routing. The study was intended to achieve the high throughput path among the source and destination and the mechanism which is presented is the test over the 23 number of nodes, and each node are placed with 802.11 wireless cards. The method has obtained the better results in routing than other existing metrics. The combined work of Iannone and Fdida [39] gives a conceptualized overview of mesh distance vector mechanism for the routing of WMN. The mechanism combines routing computation of routing along with the client's path demand for the network. The presented mechanism offers better packet data, exchange, format procedures. The method gives the reduced routing table size, easy management. A Hybrid or combined routing mechanism (Proactive and reactive algorithm) for WMN is presented in $\mathrm{Oh}$ [40]. In this, the proactive algorithm will function when the network has low mobility while the reactive algorithm will function when the network becomes vary mobile. Shih et al. [41] provided a decentralized mini slot uplink and downlink scheduling protocol traffic in IEEE 802.16. In this work, the simulation results represent better network throughput. Li et al. [42] illustrated the secure transmission protocol with efficient identity-based encryption in WMN. Author has demonstrated that the session key has the few security properties which chronicle enforceability, privacy and non-renouncement, and the new convention has the greatly improved execution than the other existing techniques.

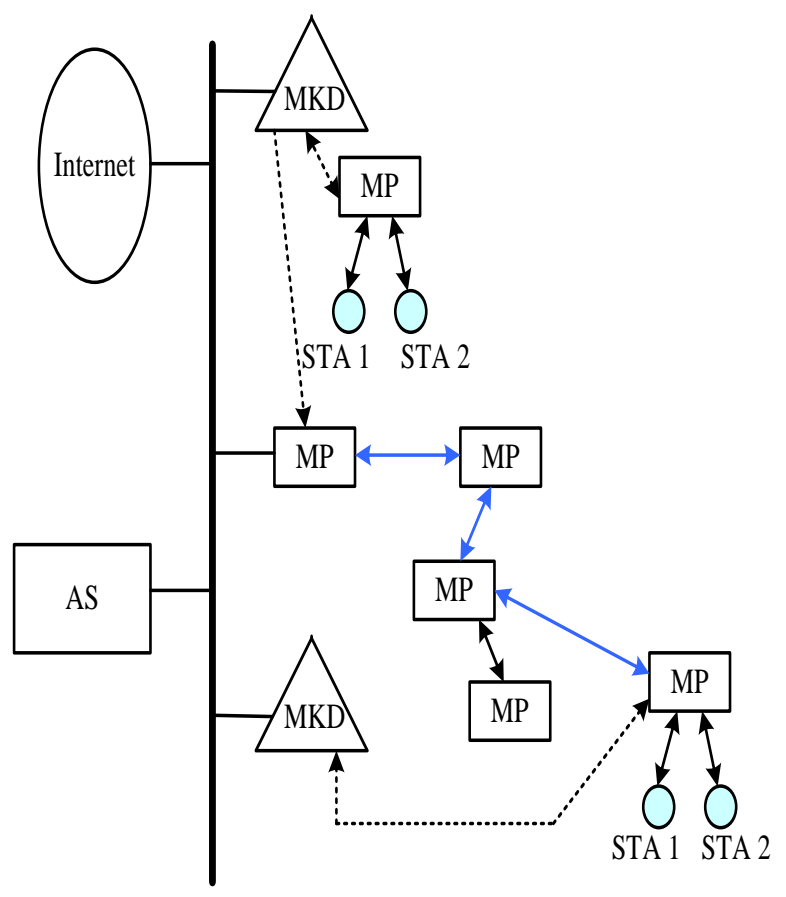

Fig. 3. Wi-Fi Mesh mechanism in Li et al. [42]

The above Fig.3 gives visualization of the Wi-Fi mesh model adopted in Li et al. [42] work. Basically, the technique has attempted to provide security over multiple hop WMN using three different modules i.e. i) mesh point, ii) key distributor, and iii) authentication server. It uses conventional identity-based encryption technique for accomplishing security features in WMN. Mogaibel et al. [43] have presented the ondemand adhoc routing protocol for the purpose of enhancing channel allocation scheme in WMN. The simulation results shows improve performance of multi-radio multichannel WMN. Majority of such forms of implementation was carried out considering simulation parameters e.g. traffic type, simulation time, propagation model (two-ray ground 
reflection), number of nodes, packet size, number of radios, traffic type, and number of connection. Boukerche et al. [44] have used Optimized Link State Routing (OLSR) in order to form a large-scale self-organized WMN. The eminent advantage of a self-organized network is its ability to perform network control and management, which reduces both the developmental complexity and the need for maintenance of these networks. The model concludes that it increases throughput and improves the delay and packet delivery of the overall network when compared to the original OLSR protocol.

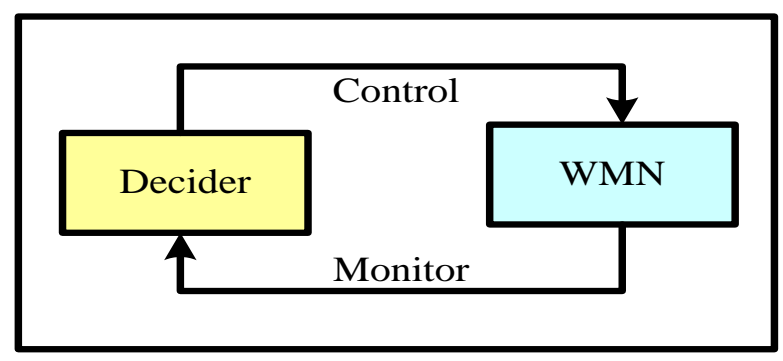

Fig. 4. The flow of [44] work

Fig.4 shows that decider flow consists of the guidelines and connected strategies for the component to be overseen (i.e., the cross section switch). Controls flow is responsible for controlling the properties of presented technique and applying them to the picked directing convention and the monitor flow will collect the data and sends it to the decider stream, Aiming to build the tenets and arrangements as occasions are produced will in the system. Tsai and Chen [45] have described an IEEE 802.11 MAC Protocol over for issues in WMN. Author has discussed his work performance by comparing with other existing research works. $\mathrm{Li}$ et al. [46] have presented a technique where the frequently used on-demand algorithm was enhanced to incorporate security feature. The study outcome shows that the presented is more effective in security against identified routing attacks. Le et al. [47] have presented a concept that computes the traffic load in presence of multiradio over WMN. The analysis of simulation results gives that the network performance was enhanced completely in multiradio mesh network. Li et al. [48] have illustrated a novel technique that can perform authentication of the wireless mesh network using Kerberos protocol. The conventional Kerberos protocol possess a few constraints in accomplishing clock synchronization and putting away key; in the interim, it is powerless from secret word speculating assault and assaults brought on by malignant programming. In this work, a very unique technique of authentication was presented by the author. By using public-key encryption strategies, the security of the proposed plan is improved. The examination demonstrates that the enhanced authentication technique is fit for remote Mesh system, which can make character confirmation more secure and productive.

Zhao et al. [49] have provided a hybrid technique of constructing communication within WMN. Network topology has been effectively looked into and created as a key answer for enhancing the execution and administrations of remote interchanges. The implementation of the presented technique was carried out over a network with fixed backbone. The authors have presented communication scheme for both Intra and inter WMN. The simulated outcome was assessed using throughput, delay, and mean queue size. In this, the hierarchical mesh network is more than that Point-toMultipoint (PMP) network. The coverage area of the hierarchy mesh network is larger than that of the PMP network, so it could support more clients and get a higher network throughput. Sun et al. [50] have given a new routing protocol in cognitive wireless mesh networks. An innovative routing protocol associated with cognitive radio named AODV-COG was presented in this work. The simulation results show improved the throughput of the network. Ding et al. [51] have provided the reputation-based Proactive Routing Protocol (PRP) for the Wireless Mesh Backbone (WMB). In this author has designed the adaptive reputation management mechanism and with its simulation results he has concluded that the routing performance was enhance.

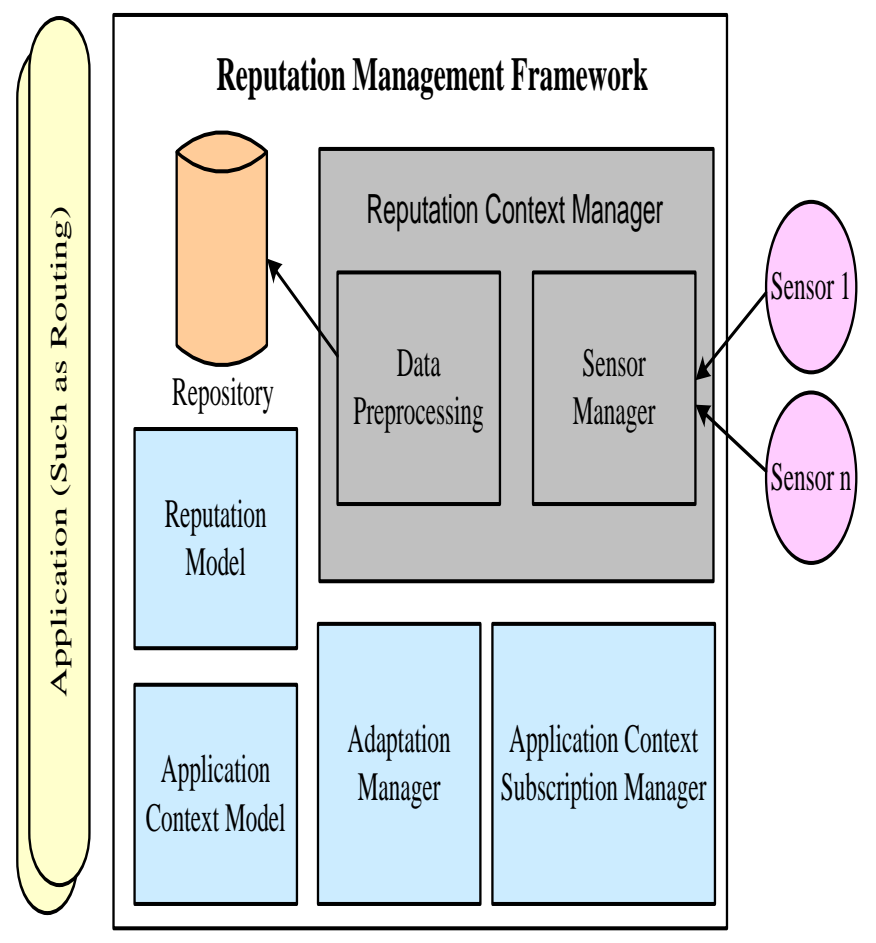

Fig. 5. Ding et al. [51]'s Adaptive Application Systems

Fig.5 represents the author's adaptive application system architecture. The architecture has context-aware applications, external source, and Adaptive Reputation Management Framework (ARMF) which computes, proliferates, stores, notoriety data gathered from heterogeneous connection sources, and makes this data accessible to abnormal state applications. For this situation, they are the routing protocol with various routing measurements. Paschoalino and Madeira [52] has demonstrated the Scalable Link Quality Routing Protocol for Multi-radio WMN. This simple and scalable approach identified the shortest paths and achieved better performance than OLSR, with a low increment in overhead. Jain et al. [53] have presented distributed protocols for WMN to schedule and control transmission rate to have Max-Min Fairness connection. In this, flow control of back pressure is used to minimize the rate at which packets are injected into the 
network to flow transmission rate not exceeding its maximum rate delivered to the fair destination rate. Matam et al. [54] have presented a security technique towards link establishment in WMN. The current peer joins Foundation convention archived in IEEE 802.11s standard is not secure and powerless against transfer and wormhole attacks. To address this issue, a proficient strategy utilizing area data is presented in this work. Security of the proposed system is examined utilizing simulation study. Wehbi et al. [55] described a client management protocol for WMN that can offer the better connection for to clients while moving while moving. In the author gives the key mechanism for upcoming WMN. AIGhadanfary and Al-Somaidai [56] have presented routing protocols and simulated some of these protocols for multimedia applications. The result of the works says that throughput performance of AODV protocol is better when network nodes increased both for moving or fixed scenarios.

\section{OPEN RESEARCH ISSUES}

After reviewing the existing techniques towards WMN, it has been seen that there are various forms of the techniques that has been introduced till date in order to enhance the communication performance of WMN. Still, there are certain pitfalls that have not been found to be addressed and hence they broaden the scope of further research work towards such unaddressed problems. The brief highlights of the open research issues are as follows:

- Less Focus towards Joint Protocol: There are lesser studies carried out towards joint protocol usage. Although, certain number of joint routing and energy problems has been addressed, but none of the studies till date has used standard RF circuitry principle of the transmitting node. For this reason, the existing techniques could show superior outcomes from simulation viewpoint but it doesn't guarantee its applicability in real-world implementation.

- Few Enhancements on Scheduling: The existing scheduling techniques are more focused on channel resources in order to obtain better quality-of-service. However, there is no focus on energy efficiency towards existing scheduling techniques that possess a big impediment towards nodes working on adverse geographical environment.

- Less Predictive Approach to Ensure QoS: Although, there are voluminous studies being carried out towards ensuring reduced delay, higher throughput, etc, but existing techniques doesn't really use any form of predictive schemes based on dynamicity of the traffic. There are also less number of studies where QoS is guaranteed over the uncertain presence of congestion factor. Existing mechanism will require prior information about the traffic load in order to ensure QoS which doesn't happens in real time.

- Less benchmarked techniques on Routing: At present, there is no standard or benchmarked modeling of any research work where optimal performance of routing is reported. Studies towards benchmarking will require extensive test-environment to implement the routing over WMN, which is yet to be explored in future.

- Few Focus on Optimization: Existing optimization of communication performance in WMN calls for using highly recursive algorithm. This causes higher depletion of node resources as well as occupancy of channel capacity. Moreover, there is no optimization techniques exist that considers network constraint, topology dynamics, and network-based problems (e.g. scattering, fading, interference, etc). Hence, study towards optimization will require a special attention.

\section{CONCLUSION}

This survey paper gives the important aspects of a Wireless Mesh Network along with some existing mechanisms in WMN routing to attain better solution for variable load. The surveys of various researches performed in WMN are discussed from recent IEEE transaction journals. With the recent research gap in existing work, the future study solution is provided which can offer a better idea to have good routing in WMN.

Our future work will be in the direction of accomplishing the open research issues. We will mainly focus on developing an effective scheduling that has potential supportability towards multihop transmission in WMN. The work will also focus on incorporating a predictive principle in order to achieve higher scalability with potential robustness against dynamic traffic condition. Finally, we will also investigate to evolve up with the new non-recursive optimization algorithm. The future solution for better routing in WMN can be attained by below strategic process.

- An algorithm can be designed to mitigate the hidden terminal issues in multi-hop WMN by reducing contention based channel access latencies.

- The optimizing algorithm for enhancing QoS parameters by considering multipath routing, congestion, load balancing.

- Benchmarking of the system by comparing the outcome will be compared along with most significant work of the existing system.

\section{REFERENCES}

[1] Lisa Gansky, The Mesh: Why the Future of Business Is Sharing, Penguin, 2010

[2] Vishram Mishra, Jimson Mathew, Lau Chiew Tong, QoS and Energy Management in Cognitive Radio Network: Case Study Approach, Springer-Technology \& Engineering, 2016

[3] Karthika K. C, "Wireless mesh network: A survey," 2016 International Conference on Wireless Communications, Signal Processing and Networking (WiSPNET), Chennai, 2016, pp. 1966-1970.

[4] M. Eslami, O. Karimi and T. Khodadadi, "A survey on wireless mesh networks: Architecture, specifications and challenges," 2014 IEEE 5th Control and System Graduate Research Colloquium, Shah Alam, 2014, pp. $219-222$

[5] D. C. Karia, A. Jadiya and R. Kapuskar, "Review of Routing Metrics for Wireless Mesh Networks," 2013 International Conference on Machine Intelligence and Research Advancement, Katra, 2013, pp. 47-52.

[6] Ian F. Akyildiz, Xudong Wang, Wireless Mesh Networks, John Wiley \& Sons, 2009

[7] A. B. M. Alim Al Islam, M. J. Islam, N. Nurain and V. Raghunathan, "Channel Assignment Techniques for Multi-Radio Wireless Mesh 
Networks: A Survey," in IEEE Communications Surveys \& Tutorials, vol. 18 , no. 2, pp. 988-1017, Secondquarter 2016.

[8] F. Giust, L. Cominardi and C. J. Bernardos, "Distributed mobility management for future 5G networks: overview and analysis of existing approaches," in IEEE Communications Magazine, vol. 53, no. 1, pp. 142-149, January 2015

[9] T. Sharma, K. Ritesh, N. Chauhan and S. Agarwal, "Analogous study of 4G and 5G," 2016 3rd International Conference on Computing for Sustainable Global Development (INDIACom), New Delhi, 2016, pp. 2137-2140.

[10] Y. L. Ban, C. Li, C. Y. D. Sim, G. Wu and K. L. Wong, "4G/5G Multiple Antennas for Future Multi-Mode Smartphone Applications," in IEEE Access, vol. 4, no. , pp. 2981-2988, 2016.

[11] V.C. Gungor1, E. Natalizio2, P. Pace3, and S. Avallone, "Challenges and Issues in Designing Architectures and Protocols for Wireless Mesh Networks", Springer Journal for Wireless Mesh Network, pp.1-27, 2008

[12] S. Kolipaka, B. N. Bhandari and A. Dey, "Joint Admission Control and vertical handoff between WLAN and WIMAX in wireless mesh networks for QoS," 2016 IEEE International Conference on Engineering and Technology (ICETECH), Coimbatore, 2016, pp. 10181023.

[13] A. V. R. Mayuri and M. V. Subramanyam, "MPGA: QOS adequacy latitude aware cooperative spectrum sensing in Cognitive Wireless Mesh Networks by Meticulous Progression based GA," 2015 Conference on Power, Control, Communication and Computational Technologies for Sustainable Growth (PCCCTSG), Kurnool, 2015, pp. 318-325.

[14] N. R. Appini and C. D. V. SubbaRao, "QoS Aware Multicast Framework based on WayPoint routing for Hybrid Wireless Mesh Networks," 2015 IEEE International Conference on Electrical, Computer and Communication Technologies (ICECCT), Coimbatore, 2015, pp. 1-8.

[15] H. Li, J. Zhang, Q. Hong, H. Zheng, Y. Wang and J. Zhang, "QoS-aware channel-width adaptation in wireless mesh networks," 2016 IEEE International Conference on Communications (ICC), Kuala Lumpur, 2016, pp. 1-6.

[16] John Edwards, The future of military comms on the battlefield, An Article from Defense System. Accessed from https://defensesystems.com/articles/2012/02/08/cover-story-militarycommunications-technologies.aspx on 31st Jan, 2017

[17] Y. Xu and W. Wang, "Wireless Mesh Network in Smart Grid: Modeling and Analysis for Time Critical Communications," in IEEE Transactions on Wireless Communications, vol. 12, no. 7, pp. 3360-3371, July 2013.

[18] https://www.google.com/patents/US9125041

[19] Raniwala, Ashish, and Tzi-cker Chiueh. "Architecture and algorithms for an IEEE 802.11-based multi-channel wireless mesh network." Proceedings IEEE 24th Annual Joint Conference of the IEEE Computer and Communications Societies.. Vol. 3. IEEE, 2005.

[20] Navda, Vishnu, Anand Kashyap, and Samir R. Das. "Design and evaluation of imesh: an infrastructure-mode wireless mesh network." Sixth IEEE International Symposium on a World of Wireless Mobile and Multimedia Networks. IEEE, 2005.

[21] Akyildiz, Ian F., and Xudong Wang. "A survey on wireless mesh networks." IEEE Communications magazine 43.9 (2005): S23-S30.

[22] Lee, K-D., and Victor CM Leung. "Fair allocation of subcarrier and power in an OFDMA wireless mesh network." IEEE Journal on Selected Areas in Communications 24.11 (2006): 2051-2060.

[23] Niculescu, Dragos, et al. "Performance of VoIP in a 802.11 Wireless Mesh Network." INFOCOM. 2006.

[24] Radunović, Božidar, et al. "Horizon: balancing TCP over multiple paths in wireless mesh network." Proceedings of the 14th ACM international conference on Mobile computing and networking. ACM, 2008.

[25] Akyildiz, Ian F., Xudong Wang, and Weilin Wang. "Wireless mesh networks: a survey." Computer networks 47.4 (2005): 445-487.

[26] Jun, Jangeun, and Mihail L. Sichitiu. "The nominal capacity of wireless mesh networks." IEEE wireless communications 10.5 (2003): 8-14.

[27] Draves, Richard, Jitendra Padhye, and Brian Zill. "Routing in multiradio, multi-hop wireless mesh networks." Proceedings of the 10th annual international conference on Mobile computing and networking. ACM, 2004.

[28] A. Al-Saadi, R. Setchi, Y. Hicks and S. M. Allen, "Routing Protocol for Heterogeneous Wireless Mesh Networks," in IEEE Transactions on Vehicular Technology, vol. 65, no. 12, pp. 9773-9786, Dec. 2016.

[29] U. Ashraf, "Capacity Augmentation in Wireless Mesh Networks," in IEEE Transactions on Mobile Computing, vol. 14, no. 7, pp. 13441354, July 12015.

[30] S. Avallone and A. Banchs, "A Channel Assignment and Routing Algorithm for Energy Harvesting Multiradio Wireless Mesh Networks," in IEEE Journal on Selected Areas in Communications, vol. 34, no. 5, pp. 1463-1476, May 2016.

[31] S. Chakraborty, S. Nandi and S. Chattopadhyay, "Alleviating Hidden and Exposed Nodes in High-Throughput Wireless Mesh Networks," in IEEE Transactions on Wireless Communications, vol. 15, no. 2, pp. 928-937, Feb. 2016.

[32] Z. M. Fadlullah, T. Nakajo, H. Nishiyama, Y. Owada, K. Hamaguchi and N. Kato, "Field measurement of an implemented solar powered BSbased wireless mesh network," in IEEE Wireless Communications, vol. 22, no. 3, pp. 137-143, June 2015.

[33] M. Li, H. Nishiyama, N. Kato, Y. Owada and K. Hamaguchi, "On the Energy-Efficient of Throughput-Based Scheme Using Renewable Energy for Wireless Mesh Networks in Disaster Area," in IEEE Transactions on Emerging Topics in Computing, vol. 3, no. 3, pp. 420431, Sept. 2015.

[34] C. C. Lin, L. Shu and D. J. Deng, "Router Node Placement With Service Priority in Wireless Mesh Networks Using Simulated Annealing With Momentum Terms," in IEEE Systems Journal, vol. 10, no. 4, pp. 14021411, Dec. 2016

[35] M. Mansoori, M. Mahdavi and H. Amini Khorasgani, "Performance Analysis of Large Multi-Interface Wireless Mesh Networks with MultiDifferent Bandwidth Channel," in IEEE Transactions on Mobile Computing, vol. 15, no. 5, pp. 1237-1248, May 12016.

[36] H. T. Roh and J. W. Lee, "Channel assignment, link scheduling, routing, and rate control for multi-channel wireless mesh networks with directional antennas," in Journal of Communications and Networks, vol. 18, no. 6, pp. 884-891, Dec. 2016.

[37] X. Yu, P. Navaratnam, K. Moessner and H. Cruickshank, "Distributed Resource Reservation in Hybrid MAC With Admission Control for Wireless Mesh Networks," in IEEE Transactions on Vehicular Technology, vol. 64, no. 12, pp. 5891-5903, Dec. 2015.

[38] Draves, Richard, Jitendra Padhye, and Brian Zill. "Routing in multiradio, multi-hop wireless mesh networks." Proceedings of the 10th annual international conference on Mobile computing and networking. $\mathrm{ACM}, 2004$.

[39] Iannone, Luigi, and Serge Fdida. "Meshdv: A distance vector mobilitytolerant routing protocol for wireless mesh networks." IEEE ICPS Workshop on Multi-hop Ad hoc Networks: from theory to reality (REALMAN). 2005.

[40] Oh, Minseok. "A hybrid routing protocol for wireless Mesh Networks." 2008 IEEE International Symposium on Broadband Multimedia Systems and Broadcasting. IEEE, 2008.

[41] Shih, Kuei-Ping, Hung-Chang Chen, and Chi-Tao Chiang. "A Decentralized Minislot Scheduling Protocol (DMSP) for uplink and downlink traffic in IEEE 802.16 wireless mesh networks." 2009 IEEE 20th International Symposium on Personal, Indoor and Mobile Radio Communications. IEEE, 2009.

[42] Li, Yahui, et al. "Efficient security transmission protocol with identitybased encryption in wireless mesh networks." High Performance Computing and Simulation (HPCS), 2010 International Conference on. IEEE, 2010.

[43] Mogaibel, Hassen Abd-Almotaleb, et al. "Channel Reservation Scheme Based on AODV Routing Protocol for Common Traffic in Wireless Mesh Network." Computer and Network Technology (ICCNT), 2010 Second International Conference on. IEEE, 2010.

[44] Boukerche, Azzedine, et al. "A self-x approach for OLSR routing protocol in large-scale wireless mesh networks." IEEE GLOBECOM 2008-2008 IEEE Global Telecommunications Conference. IEEE, 2008. 
[45] Tsai, Tzu-Jane, and Ju-Wei Chen. "IEEE 802.11 MAC protocol over wireless mesh networks: problems and perspectives." 19th International Conference on Advanced Information Networking and Applications (AINA'05) Volume 1 (AINA papers). Vol. 2. IEEE, 2005.

[46] Li, Celia, Zhuang Wang, and Cungang Yang. "SEAODV: A Security Enhanced AODV routing protocol for wireless mesh networks." Transactions on computational science XI. Springer Berlin Heidelberg, 2010. 1-16.

[47] Le, Anh-Ngoc, Dong-Won Kum, and You-Ze Cho. "Load-aware routing protocol for multi-radio wireless mesh networks." Communications and Electronics, 2008. ICCE 2008. Second International Conference on. IEEE, 2008

[48] Li, Min, et al. "A novel identity authentication scheme of wireless mesh network based on improved kerberos protocol." Distributed Computing and Applications to Business, Engineering and Science (DCABES), 2014 13th International Symposium on. IEEE, 2014.

[49] Zhao, Liqiang, et al. "A hybrid routing protocol for hierarchy wireless mesh networks." 2010 6th International Conference on Wireless Communications Networking and Mobile Computing (WiCOM). IEEE, 2010.

[50] Sun, Xuebin, Yurong Zhang, and Chenglin Zhao. "A new routing protocol in cognitive wireless mesh networks." Advanced Intelligence and Awarenss Internet (AIAI 2010), 2010 International Conference on IET, 2010.
[51] Ding, Qing, et al. "RePro: A reputation-based proactive routing protocol for the wireless mesh backbone." INC, IMS and IDC, 2009. NCM'09. Fifth International Joint Conference on. IEEE, 2009.

[52] Paschoalino, Rachel de C., and Edmundo RM Madeira. "A scalable link quality routing protocol for multi-radio wireless mesh networks." Computer Communications and Networks, 2007. ICCCN 2007. Proceedings of 16th International Conference on. IEEE, 2007.

[53] Jain, Shweta, Samir R. Das, and Himanshu Gupta. "Distributed protocols for scheduling and rate control to achieve max-min fairness in wireless mesh networks." 2007 IEEE International Symposium on a World of Wireless, Mobile and Multimedia Networks. IEEE, 2007.

[54] Matam, Rakesh, Somanath Tripathy, and Bhumireddy Swathi. "Provably secure Peer-link establishment protocol for wireless mesh networks." 2014 International Conference on Advances in Computing, Communications and Informatics (ICACCI). 2014.

[55] Wehbi, Bachar, Wissam Mallouli, and Ana Cavalli. "Light client management protocol for wireless mesh networks." 7th International Conference on Mobile Data Management (MDM'06). IEEE, 2006.

[56] Al-Ghadanfary, Karam Anan, and Mohammed Basheer Al-Somaidai. "Simulation of some Routing Protocols in a client wireless mesh network for multimedia applications." Electrical, Communication, Computer, Power, and Control Engineering (ICECCPCE), 2013 International Conference on. IEEE, 2013 